\title{
The Pharmacologic Intensification of the Water Dissociation Process, or Human Photosynthesis, and Its Effect over the Recovery Mechanisms in Tissues Affected by Bloodshed of Diverse Etiology
}

\author{
Arturo Solís Herrera, María del Carmen Arias Esparza, J. Jesús Alvarado Esquivel, Graciela Landín \\ Miranda, Ruth Isabel Solís Arias, Paola Eugenia Solís Arias, Martha Patricia Solís Arias
}

Human Photosynthesis Study Center, Centro Aguascalientes, México.

Email: comagua2000@yahoo.com

Received February 22 ${ }^{\text {nd }}, 2011$; revised March 19 ${ }^{\text {th }}, 2011$; accepted April 28 ${ }^{\text {th }}, 2011$.

\begin{abstract}
The photoreceptor layer of the human retina has several characteristics that are unique. Their energy requirements are the highest in the organism; in proportion, rods and cones require 10-fold the energy consumed by the cerebral cortex, 6-fold more than the cardiac muscle, and 3-fold more than the renal cortex. Astonishingly, the photoreceptor layer has no blood vessels at all. So, where is the energy to this tissue coming from? In this article we'll describe the hitherto unknown explanation.
\end{abstract}

Keywords: Melanin, Hydrogen, Oxygen, Water, Energy, Human Photosynthesis

\section{Background}

This paper aims to provide answers to the question: where does the energy for the human retina come from? The transduction of light by the retina uses huge amounts of energy. Current existing theories about energy require that Proteins, Lipids, and Carbohydrates must be absorbed, in principle, from ingested meals, and then they need to be transformed, in first instance, into Glucose, and then into ATP; thereafter, ATP itself seems to be the universal support for ATP energy cycles, not only in retinal cells, but in every Biological System, Known and Unknown. A very important differentiation to this respect is that one thing is the building blocks like amino acids, lipids and carbohydrates that our body absorbs from meals and thereafter uses them to form our biomass that comprises skin, nails, bones, muscle, arteries, veins, blood, etc., and another very different is the energy itself. In accordance with Dave Watson's definition, "Energy: it's something that makes things happens," in other words: Energy is defined as the ability to do work or cause change. Moreover, the anatomy and physiology of the photoreceptor layer is a good example that these functions are clearly separated.
In regards to the actual theories about the main source of energy for the human body coming from glucose, where as this compound must be transformed trough several steps into diverse compounds, since Eukaryotes do not possess functional plastids and are therefore heterotrophic: they satisfy their ATP needs through the oxidative breakdown of reduced organic compounds. Glycolysis (the Embden-Meyerhof pathway) is the backbone of eukaryotic energy metabolism: one mol glucose is oxidized to pyruvate with the help of NAD+ with a net yield of 2 mol ATP. In mitochondriate eukaryotes, pyruvate is usually further oxidized in the mitochondria through the pyruvate dehydrogenase complex $(\mathrm{PDH})$, the Krebs cycle and $\mathrm{O}_{2}$ respiration, to yield $\mathrm{CO}_{2}$ and water under the production of an additional $34-36$ mol ATP per mol glucose [1] until finally we have ATP [2]. Until this point glucose is considered as source of biomass and energy at the same time.

We know of only four basic methods to produce ATP: in the cytoplasm by photosynthesis, in chloroplasts, in bacterial cell walls, and inside the mitochondria. However, if mitochondria are the powerhouse of eukaryotic cells, which are the energy, source of the mitochondria it? According to the concept of irreducible complexity, any- 
thing less than an entire ATP molecule will not function.

The process by which ATP releases energy is poorly understood, and all living things need a constant flow of energy into and through their systems. The number of molecules of ATP synthesized by the cell, arising from glucose, it's not enough to cover the energetic requirements of the cell, given that every biochemical reaction that occurs normally in our body has energy as first requirement. Since decades ago, ATP researchers have been explaining the contradiction between energy requirements and energy availability through mechanisms such as ATP amplification; although nobody can explain it or even show it. However, the acceptations of theories like this one keep on.

However, ATP metabolic pathways continue to be in doubt. In the light of a strict chemical point of view, ATP can't fill the usual energetic and biomass requirements at the same time. On other hand, our finding of the extraordinary capacity of melanin to absorb a great part, or maybe the full electromagnetic spectrum, and the fact that with that energy melanin then splits or dissociates the water molecule, fills the gap: Energy, therefore, comes from water, and biomass comes from glucose. This astonishing explanation is congruous and coherent with the chemical and physical laws. For example: many of the chemical reactions that transfer energy in living things involve the transfer of electrons, as it happens in photosynthesis. Even more, these reactions occur most easily in the polar environment of a water solution.

Therefore the discrepancies in this process in regards to the anatomy of the human eye are also well explained: The photoreceptor layer uses 10-fold the energy requirements of the cerebral cortex, 6 times those of the cardiac muscle and 3 times more than the kidney cortex [3], and these are amazing facts because it's a layer of tissue where no blood vessel at all normally exists (see Figure 1) [4], so energy for the photoreceptors come from where?

The transport of the building blocks, or carbon chains of different lengths and orientation combined in different proportions with nitrogen, oxygen, and hydrogen that our body takes from meals by means of absorption through the gastrointestinal tract and then distributes to every cell in our organism, might take place through blood vessels and intracellular traffic itself, but the output and flow of energy must be constant as the energy that melanosomes release symmetrically in every direction.

In order for the ATP theory to work, the photoreceptor layer should have enough of a vascular network to allow an adequate supply of substances to be transporte

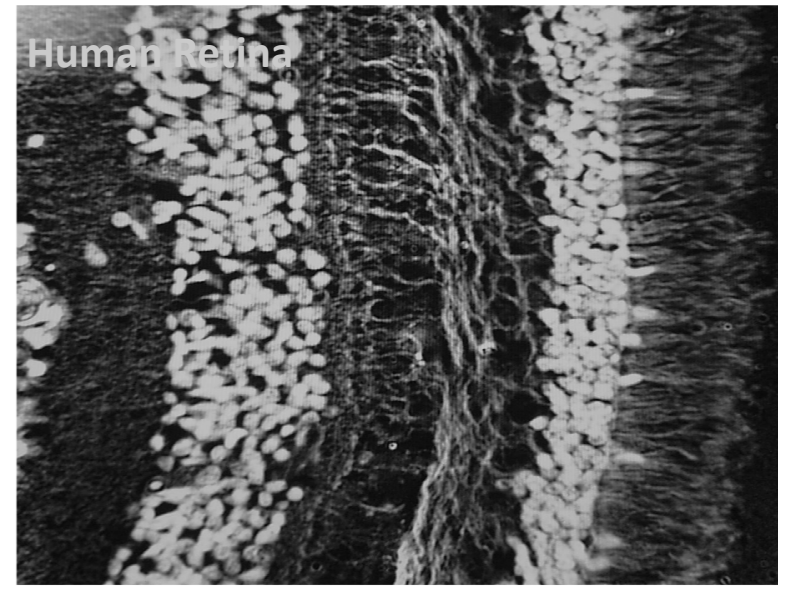

Figure 1. Human Retina, stained with $H \& E$; at right, the photoreceptor layer. (Solís-Herrera; 2005).

by the blood flow with the main aim to obtain energy. Without blood vessels a source of energy by this way is not possible. Congruously there are no blood vessels at all in the photoreceptor layer. Then the energy for the photoreceptors can't come from the blood or glucose. However, if we take into account the capacity of melanin to catch the photonic energy and transform it into chemical energy by mean of water dissociation, then there is a light at the end of the tunnel. Recall that the normal environment of any eukaryotic cell is $70 \%$ to $90 \%$ water and the photoreceptor cell it's not an exception.

\section{The Explanation}

After twelve years of studies we found a hitherto unknown explanation: There is a photo-system in human tissue, composed by Light/Melanin/Water, in the order of abundance in Nature.

And the main function of our photo-system could be represented by the next biochemical equation:

$$
2 \mathrm{H}_{2} \mathrm{O} \leftrightarrow 2 \mathrm{H}_{2}+\mathrm{O}_{2}
$$

We have named this reaction "Human Photosynthesis." Here is a brief explanation: Melanin has the amazing capacity to harvest the photonic energy of the light, visible and invisible, and then uses the energy absorbed to dissociate, split, or break the water molecule into Hydrogen and Oxygen diatomic or molecular. Of the two, the one with the most value is the Hydrogen, because is the energy carrier by excellence in the Universe, and our body is not an exception; on the other hand, Oxygen is toxic at any concentration.

The difference with chlorophyll is of paramount importance:

$$
2 \mathrm{H}_{2} \mathrm{O} \leftrightarrow 2 \mathrm{H}_{2}+\mathrm{O}_{2}
$$


In plants, the water dissociation occurs only in one direction, it's not reversible. However, in humans, the reaction will happen in both directions that mean that our photo-system could dissociate the water and then reunite it again; in other words, the Hydrogen and Oxygen could be reform into a water molecule again. The direction of the reaction depends on the reactants concentration, temperature, pressure, amount of light and other known and unknown variables.

The Human Photo-system might work night and day because melanin absorbs the full electromagnetic spectrum. Plants photo-system just works during the day due to the fact that they absorb only at 400 and 700 nanometers.

Our body begins to lose its water dissociation capability at 26 years of age, and from then on approximately $10 \%$ more each decade, and after the fifties it goes into free fall. However, the process is also sensitive to the cold, as it is in vegetables too, to iron supplements, alcohol, anti-depressants, agents used in anesthesia, pesticides, fertilizers, high fructose syrup, contaminated water, many man-made chemical compounds, to name a few factors.

Our research has shown that water dissociation was probably the very first reaction in the origin of life [5]; therefore any other biochemical process present in our body was originated or created thereafter. Therefore many diseases might respond in some degree to the pharmacologic enhancement of human photosynthesis.

In the next part of this article we will show examples of how the enhancement of human photosynthesis increases very significantly the capacity of recovery in some patients with hemorrhage in different parts of the body. By enhancing the water dissociation capability of the body, and therefore increasing the release of energy, we allow the affected tissue to drive away very adequately the deleterious effects of the bloodshed.

\section{Clinic Cases}

\subsection{Case 1}

Male patient, 20 years old, healthy, that had suffered a blunt contusion in the left eye 10 days prior, with immediate vision loss and hard pain; during the first ten days, the patient was treated with steroids, and maximal therapy to lower the intraocular pressure; with no apparent success, and while evaluating the surgical option, the patient came to our office, and we offered him treatment to enhance the human photosynthesis; the patient and his family accepted. Treatment was initiated at once at a dosage of three drops sublingually each hour. The therapeutic result was amazing (see Figure 2).

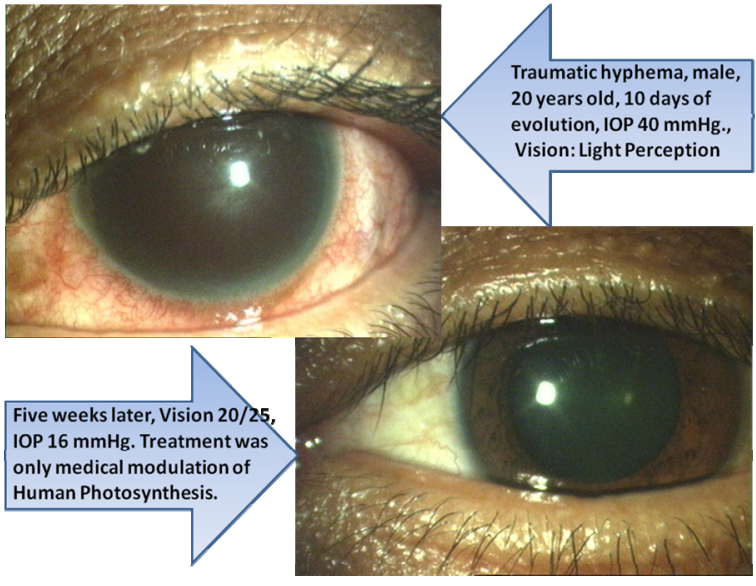

Figure 2. Notice that the bloodshed fills the anterior chamber, in spite of 10 days of maximal therapy, the bottom photography shows the recovery after 5 weeks of medical treatment.

\subsection{Case 2}

Male patient, 21 years old, healthy, with antecedents of blunt contusion in the right eye one week prior, followed by pain and vision loss. The patient was treated somewhere else with bed rest, steroids and maximal therapy in an attempt to lower intraocular pressure. And again, when the surgical option seemed like the only option to avoid corneal complications, the patient came to my office; after examination, we offered the patient treatment based on human photosynthesis enhancement; the patient and his family accepted the treatment. The dosage was three drops under the tongue each hour during the day. Results were extraordinary (see Figure 3).

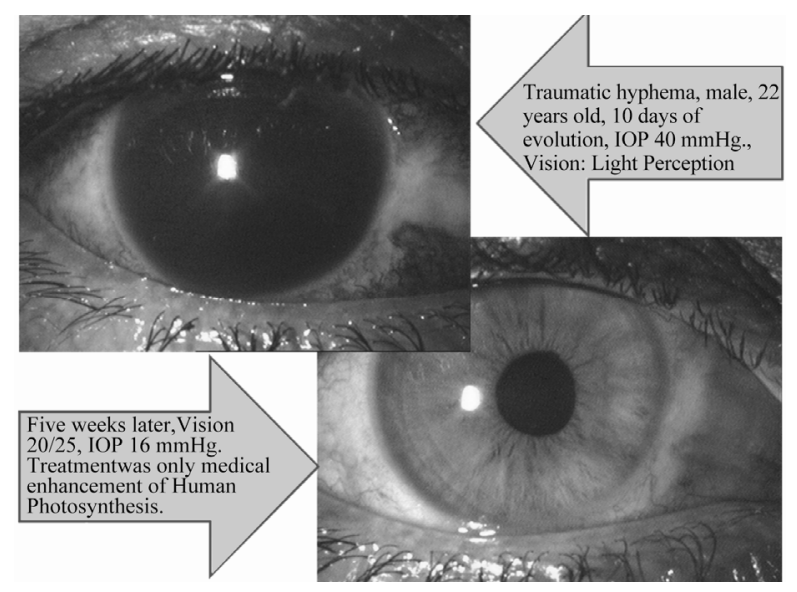

Figure 3. The bloodshed in the anterior chamber after 10 days of maximal therapy, the intraocular pressure is $\mathbf{4 0} \mathbf{m m}$ Hg. The right photography shows the extraordinary result after 5 weeks of medical therapy based in human photosynthesis enhancement. 


\subsection{Case 3}

Female patient, 64 years old, with diabetic since 1992, type 2, and with Systemic Arterial Hypertension since 1987. The patient came to see us due to sudden loss of vision in the right eye. At examination, bloodshed in the vitreous of the right eye was found. IOP was $20 \mathrm{~mm} \mathrm{Hg}$ in both eyes. The patient was treated only with Human Photosynthesis Enhancement at dosages of three drops sublingually each hour during the day (see Figure 4).

\subsection{Case 4}

Male patient, 38 years old, Diabetic with 18 years of evolution, type 1, and with Systemic Arterial Hypertension of 10 years of evolution. Six months earlier the patient had an abrupt loss of vision of the left eye by vitreous hemorrhage, treated by someone else with Laser but with no success. The patient actually came to examination due to sudden vision loss in the right eye, of 3 or 4 days of evolution, and came to the office guided by his mother. The ocular fundus showed vitreous hemorrhage that covered the macular area, and therefore the central vision was severely impaired. Treatment based in the enhancement of human photosynthesis was initiated at dosages of 3 drops sublingually each hour during the day. One week later, examination showed significant absorption of the bloodshed.

Elapsed time between each photographic register: seven days (see Figure 5).

\subsection{Case 5}

Female patient, 64 years old, married, $69 \mathrm{~kg}$ weight, 156 $\mathrm{cm}$ tall, with antecedents of ischemic cardiopathy 2 years prior, with dyslipidemia, dizziness, insomnia; dyspnea on effort, with sudden loss of consciousness a

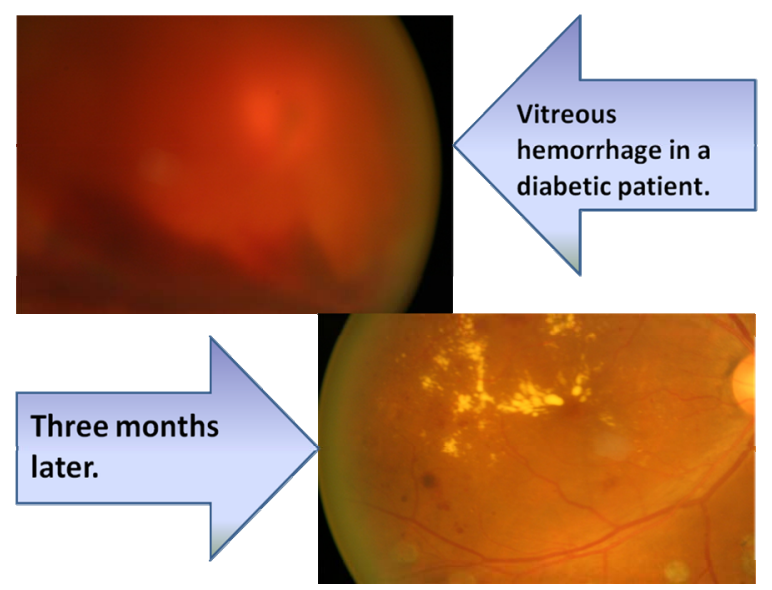

Figure 4. Photographs show therapeutic response in a diabetic female patient. Left, at first day examination, right: three months later.

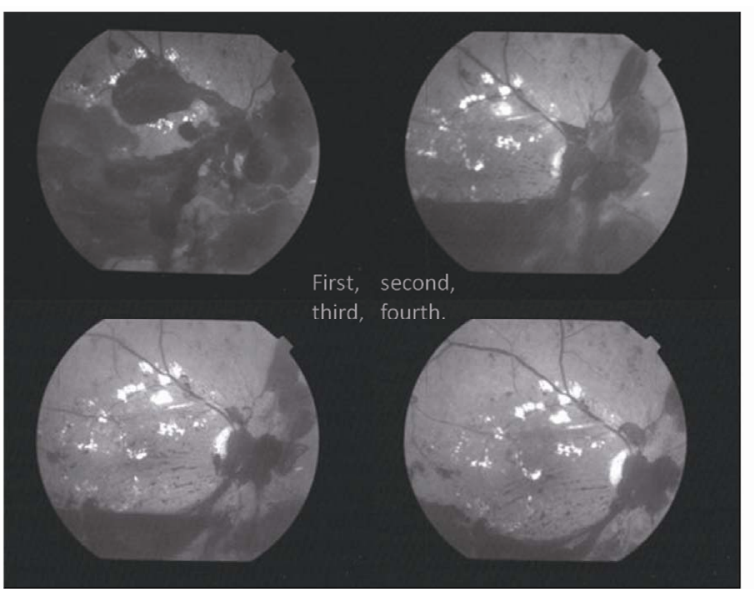

Diabetic retinopathy Treated with Human Photosynthesis Enhancement.

Figure 5. In this diabetic patient, male; with an hemorrhage that cover the macula, optic nerve and part of the posterior pole, the enhancement of human photosynthesis allows that the macula recovered function in seven days.

few hours prior; the clinical diagnosis was: Vascular Cerebral event that affected the left cerebral parenchymal at basal ganglia level and subarachnoid hemorrhage in Sylvian Area, on the left side.

A Computed tomography scan was made when she went to the emergency room, with the following results: Figures 6 and 7.

While the patient was at the ER, due to several circumstances and because the family gave their approval, the Human Photosynthesis Enhancer QIAPI 1 was administered at dosages of three drops sublingually each hour, without stopping beside common support measures; six hours later clinical symptoms had improved dramatically. Thereafter the patient continued with the QIAPI 1 treatment and was discharged with no surgery, 5 days later (see Figure 8).

A new CT scan was made 2 weeks later (8/16/2010) and the results are shown next: Figure $\mathbf{9}$.

\subsection{Comments on Case 5}

Characteristically, Human photosynthesis gets turned down with cerebral affections of diverse etiology. The brain might resist space-taking lesions, however, the main problem become the secondary reactions, such as fibrosis or gliosis. Because it's basic to cellular metabolism, it is very important to keep at the highest the water dissociation capability level to procure a fast recovery of the anatomy and physiology of the cell itself, tissues, organs, and systems. Eukaryotic cells are highly resistant if the water dissociation level is adequate. The patient went on vacation three months later to the beach at Puerto Vallarta, México. 


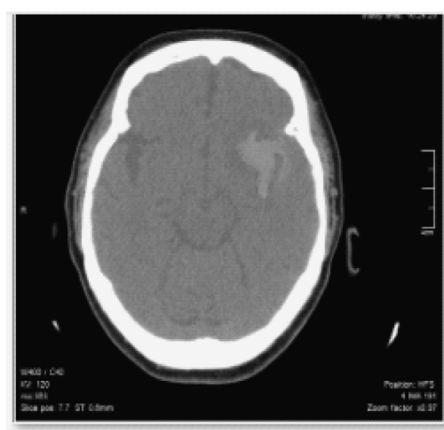

GLORIA AVELAR RIVERA--CT from 8-2-2010.SO I190

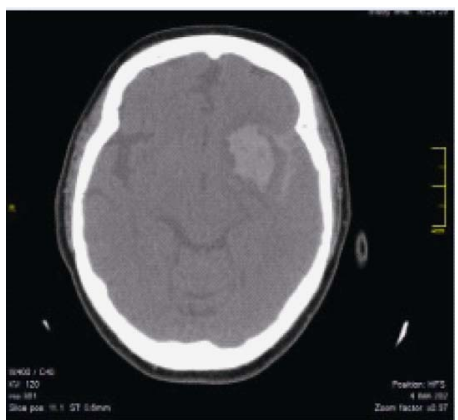

GLORIA AVELAR RIVERA--CT from 8-2-2010.SO I201

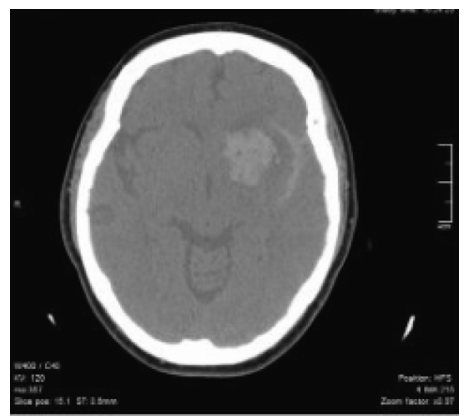

GLORIA AVELAR RIVERA--CT from 8-2-2010.SO I214

Figure 6. Study Date 8/2/2010 Images pretreatment with human photosynthesis enhancement.

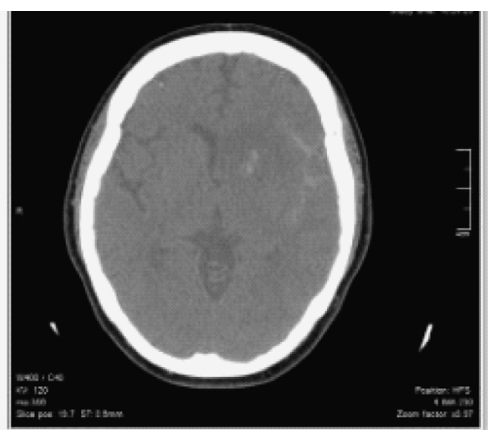

GLORIA AVELAR RIVERA--CT from 8-2-2010.SO I229

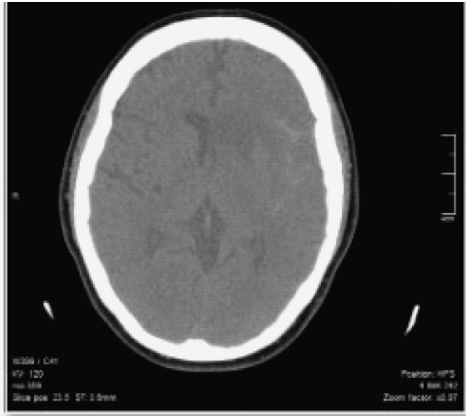

GLORIA AVELAR RIVERA--CT from 8-2-2010.SO I241

Figure 7. Study date 8/2/2010, Images pretreatment with human photosynthesis enhancement.

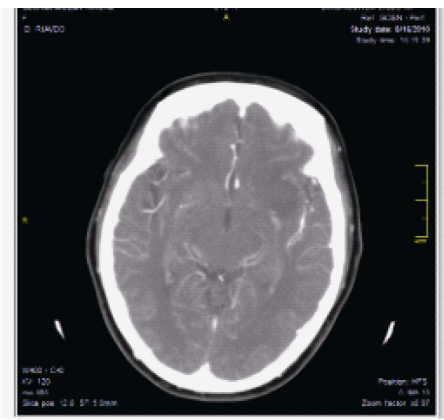

GLORIA AVELAR RIVERA--CT from 8-16-2010 C 76 SO I5

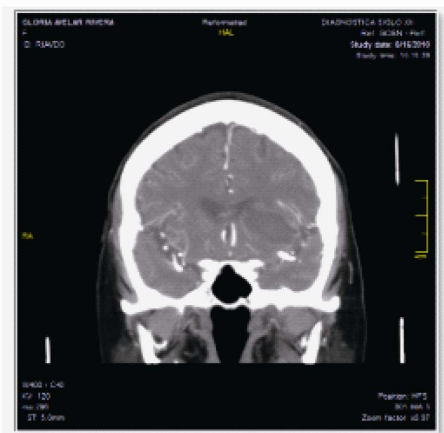

GLORIA AVELAR RIVERA--CT from 8-16-2010 C 12. SO I4

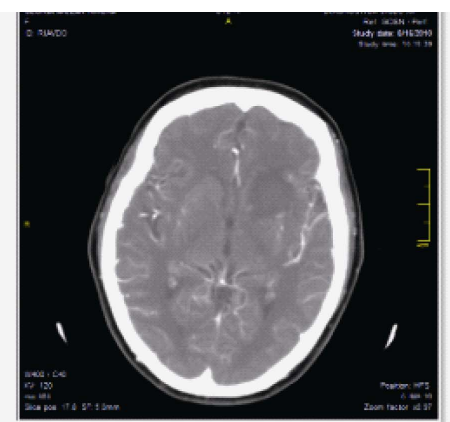

GLORIA AVELAR RIVERA--CT from 8-16-2010 C 7. SO I6

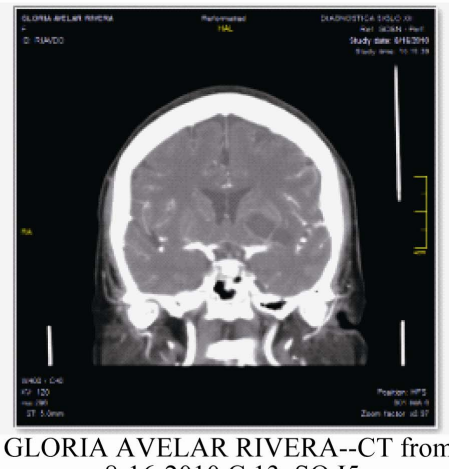
8-16-2010 C 13. SO I5

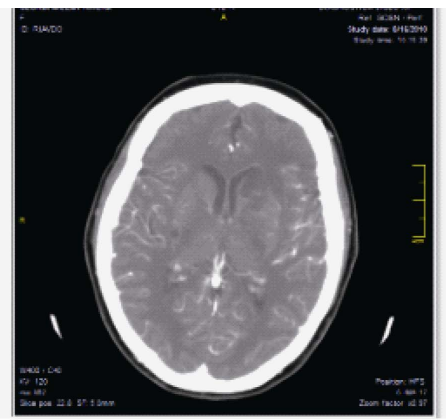

GLORIA AVELAR RIVERA--CT from 8-16-2010 C 8. SO I7

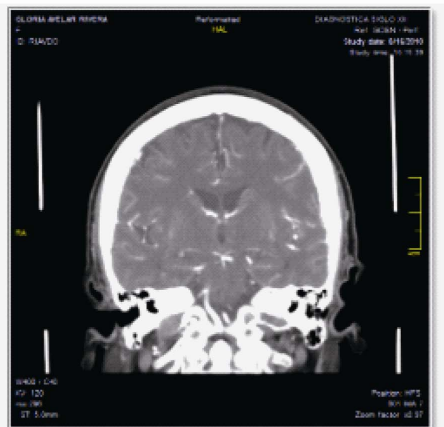

GLORIA AVELAR RIVERA--CT from 8-16-2010 C 14. SO I6

Figure 8. Study date 8/16/2010. 


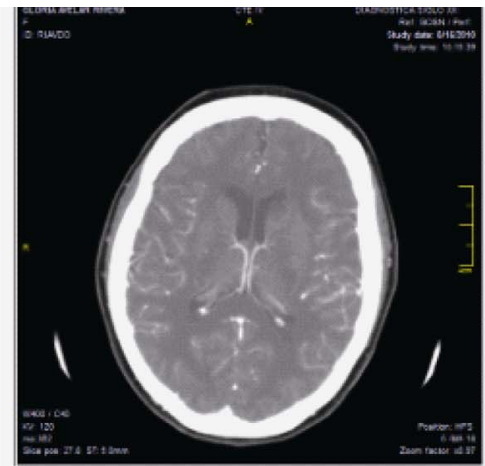

GLORIA AVELAR RIVERA--CT from 8-16-2010 C 9. SO I8

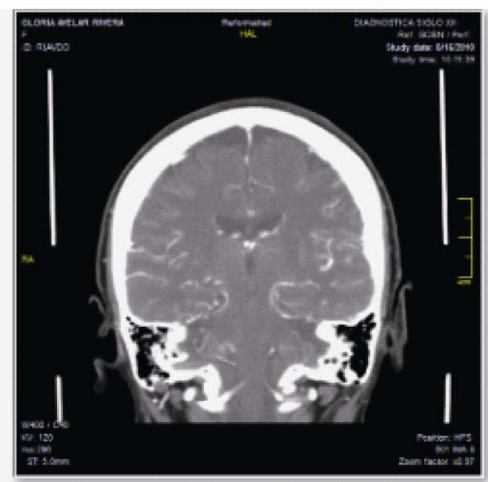

GLORIA AVELAR RIVERA--CT from 8-16-2010 C 15. SO I7

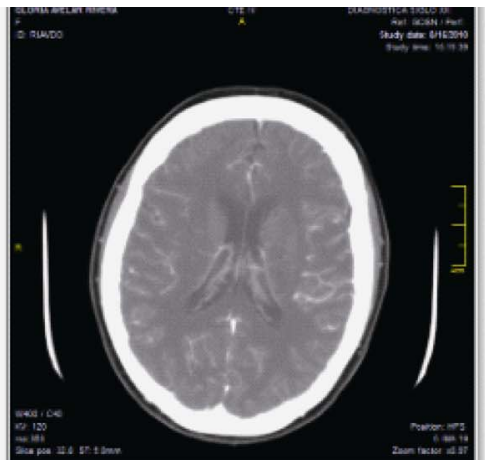

GLORIA AVELAR RIVERA--CT from 8-16-2010 C 10. SO I9

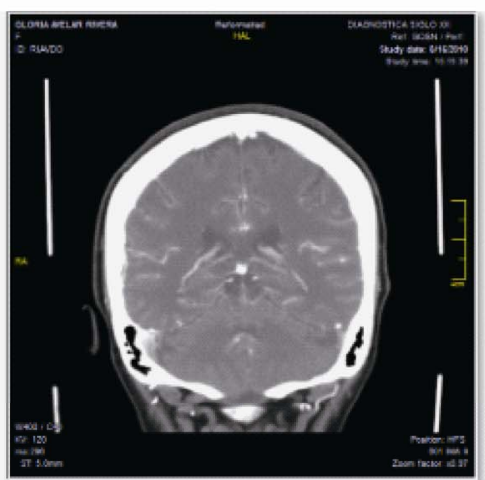

GLORIA AVELAR RIVERA--CT from 8-16-2010 C 16. SO I8

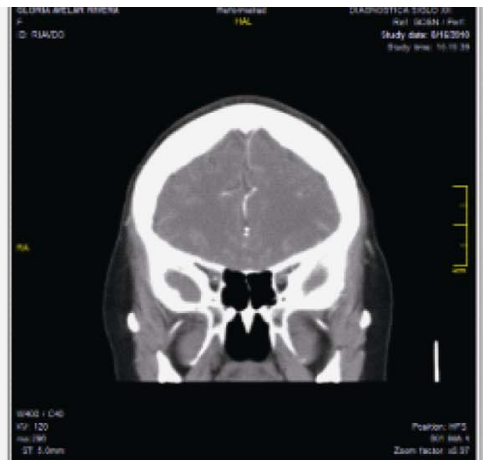

GLORIA AVELAR RIVERA--CT from 8-16-2010 C 11. SO I3

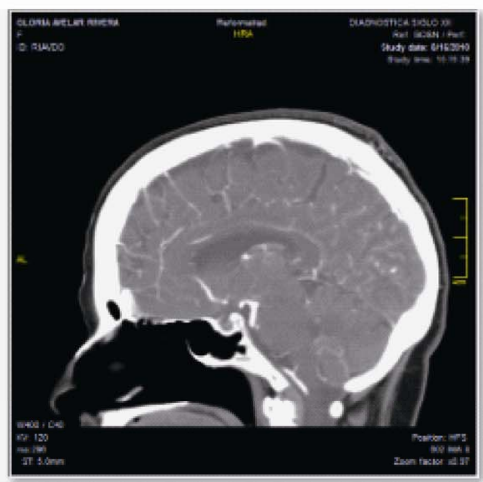

GLORIA AVELAR RIVERA--CT from 8-16-2010 C 17. SO I7

Figure 9. Study date 8/16/2010.

\subsection{Case 6}

Child, 5 years old, male; he was knocked down by a car on September 26, 2010, with loss of consciousness and coma; at the first emergency room, the coma was classified as Glasgow 2, the doctors intubated him during 7 days, and mainly due to the poor prognosis, the patient was taken to another Hospital (CMQ) on October 6 of 2010, where an CT Scan was done: Figures 10-12.

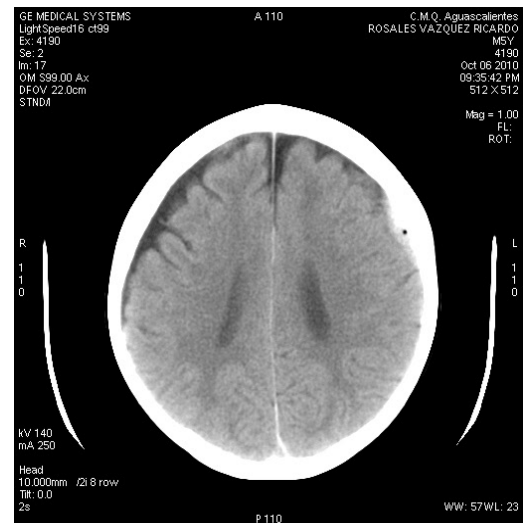

Figure 10. Acute subdural hematoma.
After the patient was moved to CMQ, QIAPI 1 was initiated at once during the first day of hospitalization, at dosages of three drops sublingually each hour and the response of the patient was dramatic; on the second day of hospitalization the Glasgow level was at 15.

This photograph (Figure 13) was taken on November 2 of 2010; the discharge from the hospital was on October 20 of 2010 .

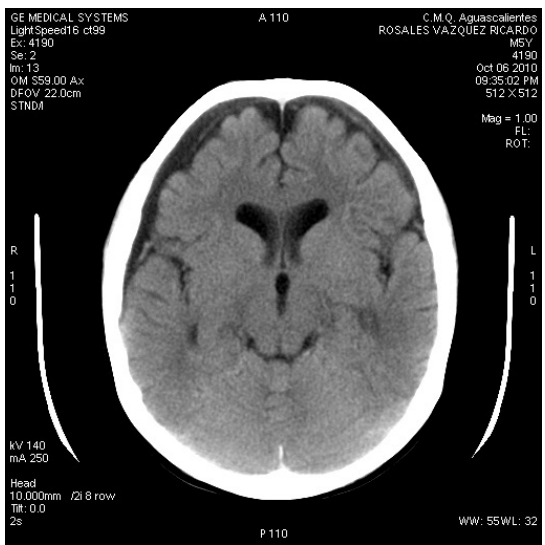

Figure 11. Chronic bilateral subdural hematomas. 


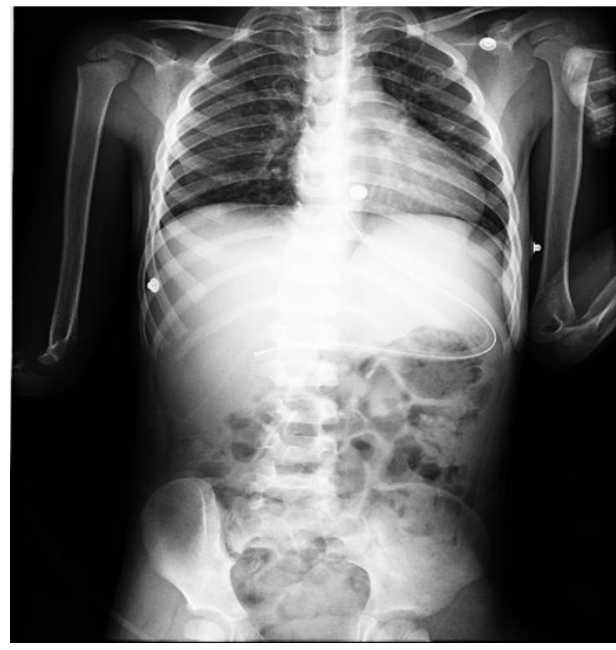

Figure 12. In $X$ rays the findings were: Pneumonia and bilateral pleural effusion. Date: Oct 6, 2010.

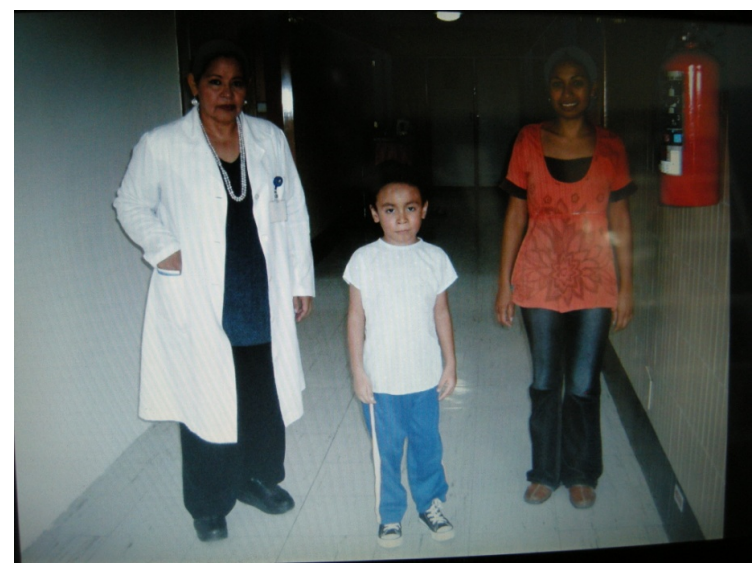

Figure 13. The patient with his mother and Dr. Landin.

\section{Conclusions}

The original spark of life may have begun in sterile conditions, otherwise it could have been instantly absorbed or devoured and the human photo-system, composed by Light/Melanin/Water, following the order of abundance in the universe, fits the Darwinian requirements.

Therefore, human photosynthesis is the origin of life and it's placed at first in the sequence of all biochemical processes of life; any other function or reaction it's secondary to water dissociation and directly or indirectly are depending of the energy released by our hitherto unknown human photo-system. More over any disease will respond to human photosynthesis enhancement in more or less degree because our organism is the result of billion's years of evolution and hence has a very fine tuning, therefore eukaryotic cell has amazing recovering capacities that allow our body to have been survived during eons of time.

In the different tissues affected by bloodshed, the tissue consistently shows a markedly recovery with no apparently sequels.

\section{REFERENCES}

[1] W. Martin and M. Müller, “The Hydrogen Hypothesis for the First Eukaryote,” Nature, Vol. 392, 1998, pp. 37-41.

[2] J. Bergman, "ATP: The Perfect Energy Currency for the Cell,” Creation Research Society Quarterly, Vol. 36, No. 1, 1999.

[3] A. Alm, “Ocular Circulation,” In: W. M. Hart Jr, Ed., Adler's Physiology of the Eye, 9th Edition, Mosby, New York, 1992, pp. 200-226.

[4] W. E. Benson, "Pathophysiology of Retinal Detachment," In: C. D. Regillo and W. E. Benson, Eds., Detachment, Diagnosis and Management, 2nd Edition, Lippincott Williams \& Wilkins, Philadelphia, 1998, pp. 17-32.

[5] A. Solís-Herrera, M. de C. Arias-Esparza, R. I. SolísArias, P. E. Solís-Arias and M. P. Solís-Arias, "The Unexpected Capacity of Melanin to Dissociate the Water Molecule Fills the Gap between the Life before and after ATP,” Biomedical Research, Vol. 21, No. 2, 2010, pp. 224-226. 